\title{
Audiodescription des arts plastiques. Autoportrait de Dora Maar
}

\author{
Audio description in the visual arts. \\ Self-portrait of Dora Maar
}

\author{
Anna Wendorff \\ Université de Łódź \\ University College London (Professeure invitée) \\ anna.wendorff@uni.lodz.pl
}

\begin{abstract}
The article addresses the problem of intersemiotic translation: translating images into words. The first part deals with the issue of image hermeneutics. Following this, eye tracking research is briefly described and museum audio description for the blind and visually impaired is introduced. A case study of Self-portrait of Dora Maar is carried out, highlighting the importance of sight in the artist's work. The text tries to answer the following questions: Who and using which techniques and strategies should translate images into words for recipients with visual disabilities, so that the translation is satisfactory and adapted to their perception? How to provide a visually impaired person with an aesthetic experience without imposing our own perception of the image on them?
\end{abstract}

Keywords: intersemiotic translation, image hermeneutics, eye-tracking, museum audio description, Dora Maar, Self-portrait

\section{INTRODUCTION}

Chaque histoire racontée est la somme des histoires de tous ceux qui en ont été témoins, elle est inhérente à l'être humain, d'autant plus à une époque où la cyberperception est devenue un état postbiologique de la société (Ascott, 1998). Qui devrait donc traduire l'image en mots, pour les personnes aveugles et malvoyantes, et cela 
à l'aide de quelles techniques et stratégies, pour que cette traduction soit satisfaisante et adaptée à leur perception ? Comment faire vivre à une personne malvoyante une expérience esthétique, sans lui imposer en même temps une perception préconçue de l'image ? L'article traite de la question de la traduction intersémiotique (Toury, 1986, p. 1114), en particulier des audiodescriptions (AD) (Chmiel \& Mazur, 2014, pp. 19-21) d'œuvres d'art (Randaccio, 2018, pp. 285-297), à l'instar d'une description du musée de Londres.

\section{ENTRE L'IMAGE ET LA PAROLE}

Afin de répondre aux questions ci-dessus, nous devons, dans un premier temps, considérer de plus près la relation entre l'image et la parole, ainsi que la traduction de l'image en mots, pour nous occuper ensuite de l'herméneutique de l'image. Il semblerait que la langue et l'image, en tant que moyens de communication, soient incompatibles, par conséquent, la traduction de ce qui est visuel n'est pas verbalement possible. Certains philosophes et historiens de l'art argumentent que l'image ne peut pas être remplacée par des mots. Selon eux, le caractère unique de la langue visuelle consiste en la mise en lumière de vérités inexprimables à l'aide d'un moyen de communication linéaire. C'est le point de vue défendu par, entre autres, Max Imdahl, créateur de l'iconique, qui estime qu'une œuvre d'art est autonome et intraduisible en discours.

L'argumentation d'Imdahl concernant l'ordre visuel repose sur le postulat selon lequel les images orientent le regard de telle manière qu'en tant que productions de connaissance autonomes, elles se présentent comme un « vecteur de sens » « qui ne peut être remplacé par quoi que ce soit d'autre ». Par « la proposition visuelle» d'une « structure de sens iconique qu'on ne peut trouver en dehors de l'image », les images apprennent à mettre en relation un regard reconnaissant (l'identification au niveau des objets représentés) avec un regard regardant (une vision détachée du niveau des objets) (Fliescher, 2017, p. 45).

L'image est, sans aucun doute, différente du texte et n'est pas son équivalent fidèle. Pourtant, la description discursive d'une œuvre d'art constitue le seul moyen de communication accessible permettant l'analyse de la langue « visuelle ». En effet, il n'existe aucune herméneutique extralinguistique, or, dans le cas de l'herméneutique de l'image, il s'agit de la traduction de cette dernière en mots, ce qui est lié indissolublement au caractère linguistique et fondamental de la réflexion sur l'art : « (...) les phénomènes purement visuels doivent être décrits, ils sont liés au langage grâce au potentiel déictique qui rend possible la communication en dehors de la langue et de l'image » (Boehm, 2014, p. 140).

Selon Gottfried Boehm (2014), l'herméneutique de l'image « prend sa source là où l'expérience visuelle de l'image se transforme en moyen de communication linguistique » (p. 144). Néanmoins, nous ne pouvons pas parler d'une traductibilité 
complète de l'image en mots, nous ne pouvons que nous approcher d'elle. D'après cet historien de l'art, la signification sensorielle de l'image (sa forme caractéristique de l'expérience sensorielle) demeure intraduisible (Boehm, 2014, pp. 234238). La réflexion sur l'image (auto-référence) mène à un conflit esthétique, un conflit de différence iconique (ce qui fait de l'image une image et non pas un énoncé), dans lequel la perception sensorielle s'oppose à la conceptualisation (Boehm, 2014, pp. 40-41).

La relation et le contraste entre la simultanéité et la successivité de l'image lui attribuent sa signification sensorielle, car seul l'œil est capable de repérer le chemin entre les éléments d'un ensemble, puis de suivre ce parcours d'une manière créative. Le trajet ne conduit pas en général, à un ensemble synthétique, il n'est pas question de la conceptualisation des notions mais d'une activité. Comme l'explique Boehm (2014) « l'œil peut, voire doit mettre en jeu son équipage corporel, il conçoit la différence iconique avec sa propre énergie, et ses traces, voies et trajectoires créent un modèle de références qui évoquent le sens, ainsi que des représentations, des éléments reconnaissables, ayant tendance à s'émanciper » (p. 238).

\section{OCULOMÉTRIE}

Les trajectoires de l'œil constituaient un domaine de recherches techniques déjà dans les années 1880 qui ont vu naître l'oculométrie, c'est-à-dire l'examen des mouvements oculaires lors de la réception de différentes communications visuelles, qui, à l'époque, se faisait via l'observation directe, c'est-à-dire l'observation à l'œil nu. Actuellement, des eyetrackers sophistiqués sont utilisés.

L'oculométrie est de nos jours la principale méthode de recherche de base pour examiner l'attention visuelle et ses applications sont utilisées dans des contextes différents, p. ex. la perception de l'art (Krejtz, Duchowski, Krejtz, Szarkowska \& Kopacz, 2016, pp. 11-20) ou l'audiodescription dans les musées (Szarkowska, Jankowska, Krejtz \& Kowalski, 2016, pp. 301-320). L'un des éléments soumis à l'analyse est la trajectoire des mouvements oculaires du visiteur d'un musée. Sur la base des recherches sur l'attention visuelle, il est possible d'analyser l'implication du visiteur et son traitement cognitif, de déterminer l'ordre de fixation sur des détails ainsi que sa durée (cartes de fixations et cartes thermiques). Les résultats obtenus peuvent servir à la traduction des images en mots au service des personnes aveugles et malvoyantes en vue de diriger leur attention d'une manière plus efficace.

Il nous semble important de rappeler que c'était Guy Thomas Buswell, l'inventeur du premier eyetracker non invasif, qui en 1935, en se servant d'un oculomètre, a entrepris les premières recherches portant sur la perception de l'art. Les résultats de ses recherches se trouvent dans How people look at pictures: a study of the psychology of perception in art. De ses analyses résulte que mieux on connaît la scène visuelle, plus 
le temps de fixation est long ${ }^{1}$ et plus sont courtes les saccades oculaires ${ }^{2}$, de même que la reconnaissance d'une scène visuelle est liée au nombre de fixations beaucoup plus restreint que durant sa mémorisation. Le plus grand nombre de points de fixation se trouve aux endroits essentiels pour la compréhension de la scène.

Buswell a également prouvé que lorsqu'on regarde un tableau, une attention particulière est accordée aux visages et aux yeux, contrairement aux autres objets et à l'arrière-plan. Il a démontré aussi que les yeux des personnes dont l'expérience personnelle est comparable regardent le même tableau avec des trajectoires similaires, et que la perception visuelle des enfants est complètement différente de celle des adultes. En outre, il a constaté que regarder des objets inconnus a un impact considérable sur le nombre plus important de mouvements oculaires et l'aspect chaotique des trajectoires des yeux. Quant aux objets concrets et aux autres activités perceptives, connus du récepteur, les mouvements oculaires se simplifient : ils durent plus longtemps et la répétitivité des lieux visualisés sur la peinture est stabilisée (les points de fixation).

Les études modernes sur l'eyetracking confirment que les profanes et les experts regardent différemment une œuvre d'art. Les experts en art scannent le tableau à la recherche de motifs et de formes et l'examinent longuement. Les profanes non entraînés se concentrent sur des objets reconnaissables et ne passent que quelques secondes devant le tableau (Koide, Kubo, Nishida, Shibata \& Ikeda, 2015 ; Kołodziej, Majkowski, Francuz, Rak \& Augustynowicz, 2018). Ce qui nous amène à constater que, d'un côté, la personne qui est capable de mieux décrire un tableau serait un historien de l'art ou l'employé d'un musée. De l'autre côté, la description d'un profane serait plus proche de celle d'un autre visiteur non initié. Surtout qu'au fil des années, les musées ont changé, de même que leurs fonctions et leur public. Dans le passé, les musées étaient principalement fréquentés par des connaisseurs, aujourd'hui leur public est diversifié, du profane absolu au spécialiste de haut niveau (Taylor \& Perego, 2021, pp. 34-35). Il faudrait mentionner la création de l'application Be My Eyes grâce à laquelle des volontaires, pouvant même ne posséder aucune connaissance dans le domaine d'études concerné, facilitent le traitement de l'information visuelle aux personnes malvoyantes et aveugles (à travers les visioconférences), dans différents contextes, y compris au musée.

Des chercheurs de l'université Queen Mary de Londres ont également démontré que les femmes scannent et regardent un tableau différemment des hommes. Ils ont procédé à un examen de suivi des yeux sur environ 400 participants au Musée des Sciences de Londres. Il s'est avéré que les femmes regardaient davantage le côté gauche du visage et l'œil gauche sur l'image, et qu'elles regardaient également le visage beaucoup plus souvent que les hommes. L'équipe a observé qu'il était possible

\footnotetext{
${ }^{1}$ La position du globe oculaire est relativement stable, pendant ce temps nous pouvons observer de petites vibrations.

${ }^{2}$ Les mouvements oculaires sautillants impliquant un déplacement très rapide du foyer de la vision d'un endroit à un autre.
} 
de déterminer le sexe du participant sur la base du modèle de balayage du tableau avec une précision de près de $74 \%$ (Coutrot, Binetti, Harrison, Mareschal \& Johnston, 2016). On pourrait donc envisager de concevoir deux versions d'AD : une, destinée aux femmes, et l'autre, aux hommes. En pratique, c'est une entreprise trop longue et trop coûteuse. Il nous semble pourtant intéressant d'examiner cet aspect de plus près, en vue d'élaborer des AD mieux adaptées au public. Dans ce cas, il faudrait comparer les deux versions pour pouvoir constater si elles sont différentes, et si les résultats sont conformes aux résultats des recherches sur la perception de l'art.

\section{AD DE L'AUTOPORTRAIT DE DORA MAAR}

Dans cette section, nous allons essayer de définir le terme « audiodescription ». $\mathrm{AD}$ est une description verbale et sonore de l'image et du contenu visuel, qui permet aux personnes aveugles et malvoyantes de s'informer sur le patrimoine culturel visuel dans le domaine des beaux-arts, des arts du spectacle ou de la cinématographie. AD dans l'espace muséal peut prendre la forme de fichiers sonores enregistrés dans un audioguide, un guide multimédia, un iPod, etc. et/ou placés sur le site web d'une institution donnée, ou peut être lue/prononcée en direct (avec ou sans script) lors d'une visite virtuelle ou réelle au musée. La visite guidée pour les malvoyants est l'une des façons les plus populaires de les impliquer dans la découverte de la collection du musée. Une telle visite en compagnie d'un guide enthousiaste et compétent constitue une opportunité exceptionnelle de poser des questions, de toucher une pièce d'exposition ou sa réplique et de créer des liens sociaux avec le groupe de visiteurs.

Dans chaque pays, il existe certaines normes pour la création de l'AD. En Angleterre, deux organisations méritent d'être mentionnées : Mind's Eye (communication personnelle, 25 février 2020) et VocalEyes (Audio Description and Access for Blind and Partially Sighed Visitors. Training manual for museum staff). Pour illustrer les instructions britanniques, nous allons nous référer à l'AD du tableau Autoportrait de l'exposition DORA MAAR présentée au musée Tate Modern à Londres, en 2019/2020.

Henriette Théodore Markovitch (1907-1997), connue sous le nom de Dora Maar, était une photographe, peintre et poète française. Représentante du courant surréaliste, elle entretenait des relations professionnelles avec beaucoup de ces figures éminentes : André Breton, Brassaï, Henri Cartier-Bresson ou Man Ray. Malheureusement, elle a subi dans les années 40 toute une série de traumatismes : son père part pour l'Argentine, sa mère et sa meilleure amie Nusch Eluard meurent subitement, sa relation avec Pablo Picasso prend fin et ses amis s'exilent. En 1945, l'artiste fait une dépression nerveuse et subit une thérapie par électrochocs.

Les difficultés qu'elle doit affronter se reflètent dans quelques-unes de ses œuvres de l'époque, entre autres dans Autoportrait. À titre d'exemple, nous voulons présenter 
l'AD de ce tableau dont l'auteure est Caroline Dawson, historienne de l'art et consultante en accessibilité et inclusion.

The painting I am going to describe is called Untitled (Self-portrait). It was made in 1945 and is an oil painting on paper and board. The painting measures $30 \mathrm{~cm}$ in height, by $22 \mathrm{~cm}$ in width. Which is around the size of an A4 piece of paper. It has been very intricately framed. At first the painting looked to me like a small canvas, but it is actually a thin piece of canvas-like paper, inserted into a tight fitting glass surround... almost like a folder. This is mounted within a gold framed insert, which is surrounded by thick mounting canvas, within a thick tarnished black and brass frame. The frame measures $69 \mathrm{~cm}$ in height and $56 \mathrm{~cm}$ in width. Along the bottom line of the frame is a gold (in colour) label, with the artists name and life dates carved into it. This is a self-portrait in its most intense form. Maar has painted only her face... it fills the rectangular painting... leaving little room at the top and bottom. Maar's portrait looks straight at us, the viewer, with a deep sadness expressed in the eyes. Even her skin looks drab and tired... worn out. Her forehead fills the top third of the painting, her eyes and nose - the middle, and her lips and chin at the bottom. Maar's dark brown hair is tied tightly behind her head... the brushstrokes showing that her hair is scraped back off her head.

Her forehead, and the left side of her face are painted as if they are in shadow... murky brown strokes of paint portray the contours of her face... detailed with light green patches. The right side of her face is lighter in colour... with greys smeared around her right eye and pinky tones across her cheeks and chin. Water has been applied to the paint below this eye. I'm not sure if I'm being over sensitive, but I feel as if this represents tears. Her straight thin lips are a meaty red, with a black line drawn between the join of her lips. Her nose cuts a dramatic vertical line down the centre of her face. It is the thinnest nose I have ever seen. Her nostrils and the bow of her upper lip create an arrow pointing to her lips below them. Streaks of dark puddle brown highlight how completely straight her nose is... the streaks are accentuated with touches of brilliant white paint. Maar's eyebrows are thin, quick black marks darting above her eyes. Her eyes themselves are to me the main focus of the painting. Her amber coloured irises are shadowed in rings of dark colouring. Dark brown, Dark grey, Dark green. As I mentioned earlier... they carry a deep sadness ${ }^{3}$ (Dawson, 2020).

Au début de la description, le titre de l'œuvre est présenté, suivi de l'année de sa parution et des techniques de peinture employées. Un autre élément de l'audiodescription est la dimension de l'image en centimètres, ici Dawson s'appuie sur les directives de Mind's Eye : les systèmes de mesures (« $30 \mathrm{~cm}$ in height, by $22 \mathrm{~cm}$ in width ») ainsi que sur celles de VocalEyes qui compare la dimension avec quelque chose de connu, par exemple une feuille de papier A4 (« around the size of an A4 piece of paper »). Cette démarche est conforme aux observations du pionnier de l'audiodescription, Joel Snyder, qui a proposé, par exemple, de comparer la hauteur du Washington Monument à cinquante éléphants posés les uns sur les autres (Snyder, 2007, p. 102), ce qui

\footnotetext{
${ }^{3}$ Les mots en gras marqués par l'auteure de l'article.
} 
semble une bonne solution, car les préférences des personnes handicapées peuvent varier par rapport à leur déficience visuelle ${ }^{4}$.

Il est important de souligner que l'auteure de l'AD a décrit le cadre dans lequel se trouve le tableau (" mounted within a gold framed insert ») et l'encadrement qui l'entoure ( « a thick tarnished black and brass frame »), ce qui est particulièrement important pour les personnes aveugles. Elles ont besoin d'une vue d'ensemble pour y introduire leur propre image en reliant les éléments successifs ${ }^{5}$.

Ensuite, l'auteure passe à la description du contenu du tableau en commençant par l'information la plus générale, conformément aux directives des organisations britanniques. En outre, Martin Heidegger, Hans-Georg Gadamer et Paul Ricœur ont formulé des démarches similaires, en soulignant que l'on ne peut comprendre le tout sans comprendre le détail, et que le détail ne peut pas être compris sans se référer à l'ensemble, en prenant comme point de repère le cercle herméneutique, c'est-à-dire une figure décrivant la manière de comprendre et d'interpréter le texte/l'image. Par conséquent, une interprétation efficace de l'œuvre est basée sur un mouvement circulaire ; du tout au détail et du détail au tout (Sochoń, 1995, p. 22). C'est pourquoi, grâce à l'AD, nous apprenons que le visage constitue l'élément unique de l'autoportrait, ce qui nous impose l'ordre logique de la description des détails selon deux schémas : I. le haut (le front), le milieu (les yeux et le nez), le bas (la bouche et le menton) ; II. les parties gauche et droite du visage, ce qui est lié à la répartition du clair-obscur et de la palette de couleurs. VocalEyes précise qu'il faut seulement éviter de sauter d'un côté à l'autre de l'image et vice-versa pour éviter que la personne malvoyante se sente perdue, car, comme nous l'avons déjà mentionné, cette dernière doit imaginer l'ensemble à partir de fragments dans son esprit, ce qui exige une grande concentration. Pour la même raison, nous devons hiérarchiser les informations afin de ne pas inonder le visiteur du musée de trop de détails, en ne décrivant que ce qui est important pour une œuvre donnée.

L'AD, remplie de couleurs, surtout sombres (le noir, le marron) qui constituent le reflet d'une vie pleine d'inquiétudes et de traumatismes, crée une ambiance lugubre et met en valeur le message que transmet le tableau : une profonde tristesse (« a deep sadness »). Mind's Eye et VocalEyes indiquent que les couleurs doivent être décrites, car la plupart des personnes aveugles ou malvoyantes sont nées avec un certain degré de déficience visuelle, par conséquent, elles ont conservé la mémoire des couleurs, et de leur côté, les aveugles de naissance, comprennent la signification culturelle de la couleur. Il peut également être utile de décrire les couleurs en termes d'expérience tactile, par exemple un bleu clair délicat. VocalEyes ajoute également que pour qu'une personne avec une déficience visuelle puisse vivre une expérience esthétique, il convient

\footnotetext{
${ }^{4}$ Dans l'audioguide du musée, une solution pratique peut consister à créer différentes options que le visiteur peut choisir (par exemple, s'il préfère que la distance soit décrite en pas ou en mètres, etc.).

${ }^{5}$ Les malvoyants peuvent voir l'image de très près, utiliser des loupes de musée ou des applications de grossissement sur leur smartphone ou leur tablette.
} 
de lui parler de l'ambiance que crée le tableau - par exemple le mystère ou la menace, en fournissant des détails visuels qui stimulent cette ambiance en vue de la faire régner.

De surcroît, afin d'assurer le plein accès à l'expérience muséale, l'AD devrait prendre en compte non seulement l'acquisition d'informations visuelles, mais aussi les éléments sociaux, cognitifs et émotionnels de la visite (Hutchinson \& Eardley, 2018, pp. 42-57). Ainsi, l'historienne de l'art raconte son histoire personnelle de la découverte du tableau (« looked to me like a small canvas, but it is actually a thin piece of canvas-like paper »), parle de ses sentiments («Her eyes themselves are to me the main focus of the painting »), des impressions que le tableau éveille en elle (« a self-portrait in its most intense form »), comme si elle parlait à un ami (« the thinnest nose I have ever seen »). En même temps, la description n'est pas dépourvue de sens de l'humour.

Il faut noter cependant que Dawson essaye de ne pas imposer son opinion ou son interprétation du tableau : «I'm not sure if I'm being over sensitive, but I feel as if this represents tears ». VocalEyes remarque qu'il est recommandé de décrire ce qui nous a permis de tirer des conclusions au lieu de les suggérer aux visiteurs handicapés qui devraient les formuler eux-mêmes (par exemple, au lieu de dire « le portrait d'une femme riche », nous devrions plutôt décrire ce qui nous permet de tirer une telle conclusion, la matière de ses vêtements ou les objets qui l'entourent, etc.).

La pertinence du message est également très importante, car l'AD n'est qu'une description orale ${ }^{6}$ et doit présenter les caractéristiques du langage parlé afin d'établir un contact aussi complet que possible avec le destinataire et de l'influencer, d'où des expressions telles que : "looks straight at us, the viewer». La fonction esthétique de la description est également renforcée par l'utilisation de répétitions : « sombre » ( « ... dark colouring. Dark brown, Dark grey, Dark green ») et « profonde tristesse » ( " with a deep sadness expressed in the eyes », " they carry a deep sadness »), qui ajoutent un caractère littéraire à la description. En construisant l'expérience esthétique, Dawson prête attention au vocabulaire qui est vif, varié et facile à retenir et évoque des impressions multisensorielles (Neves, 2012). Remarquons que l'AD de Dawson prend en considération les conseils du photographe français Paul Almasy, qui « dénonce cinq erreurs à éviter lors de la confection d'images à vocation communicative : ambiguité sémantique, absence d'échelle, vocabulaire inconnu du lecteur, contradiction entre les composants et valeur esthétique dominante » (Vanmalderen, 1982, p. 16).

Dora Maar a dédié Autoportrait au Dr Baron, spécialiste en neuro-ophtalmologie, domaine qui étudie l'interaction entre les yeux, le cerveau et les nerfs, dont elle a été la patiente vers 1945 (dans le coin inférieur gauche de l'image, à la plume bleue : «Pour le Docteur/Baron/avec l'amitié Dora Maar»). En outre, comme l'ont montré nos conversations avec les coordinateurs de l'accessibilité et les historiens de l'art à Londres, d'après sa façon de peindre, de dessiner, de construire les personnages, etc. on peut deviner que Maar souffrait de problèmes oculaires.

\footnotetext{
${ }^{6}$ Cette audiodescription a été présentée par Dawson en direct pendant une visite au musée.
} 
Les photographies sociales et documentaires (photographies « intéressées ») de l'artiste, font également partie de l'exposition à la Tate Modern. Elles sont consacrées à des personnes handicapées défavorisées, souffrant de précarité, entre autres des musiciens aveugles ou des vendeurs de rue à Barcelone (p. ex. Mendiant aveugle ou Orchestre de quatre musiciens de rue aveugles, 1934). Les portraits de personnes aveugles étaient particulièrement importants pour Maar : elle les a tous rassemblés en un photomontage intitulé Aveugles à Versailles (1936). L'intérêt que Maar porte à photographier des aveugles, ainsi que des personnes aux yeux fermés ou levés vers le ciel, suggère également le motif surréaliste du regard intérieur ; il s'inscrit parfaitement dans l'iconographie surréaliste et ses intérêts tels que le rêve, l'œil ou le subconscient.

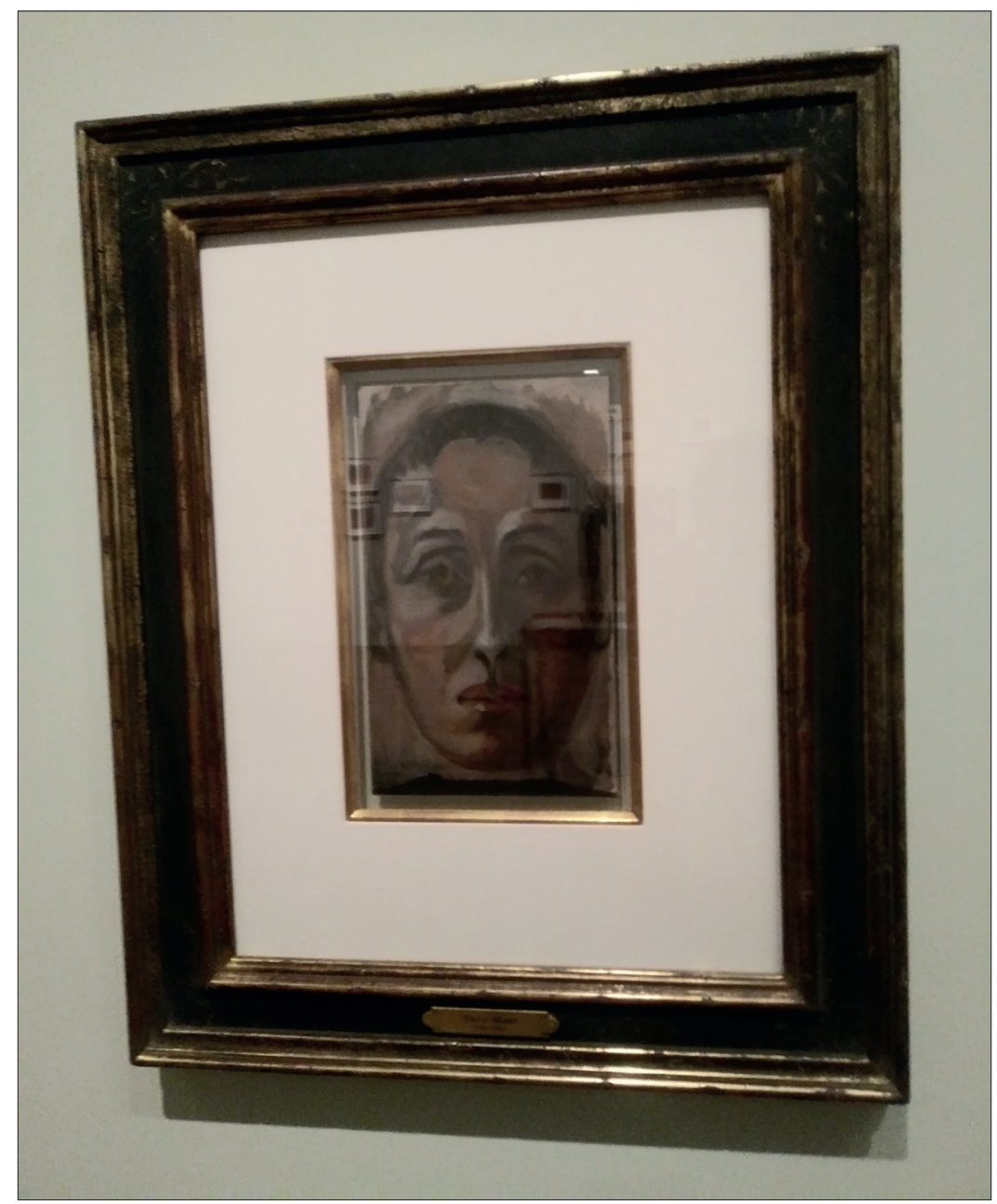

Fig. 1. Dora Maar. Autoportrait, vers 1945. Huile sur carton d'emballage Marcel Rochas. Tate Modern, Londres, février 2020, phot. Anna Wendorff 


\section{CONCLUSION}

L'audiodescription de l'image - par rapport à d'autres $\mathrm{AD}$, par exemple cinématographiques ou théâtrales - est dépourvue de canaux de communication supplémentaires tels que : dialogues, musique ou bande sonore (Mazur, 2020, p. 231), ce qui fait qu'un visiteur de musée n'obtient d'informations sur le tableau que grâce aux mots utilisés dans l'AD. D'une part, une telle description ne peut être dépourvue de toute émotion, car dans ce cas, elle ne remplirait pas sa fonction esthétique, priverait le visiteur du musée des impressions inaliénables dans la découverte de l'art, elle ne serait qu'une description aride et mathématique. D'autre part, ces impressions et sensations au contact d'une œuvre d'art doivent être décrites de manière habile afin de ne pas imposer une interprétation unique et concrète d'une image donnée (Hutchinson, 2020).

Pour répondre à la question : qui devrait préparer les audiodescriptions des arts plastiques? Il peut s'agir d'un coordinateur d'accessibilité au musée, d'un historien de l'art, d'un employé de musée formé à la conception et à l'animation de visites adaptées (c'est le cas de Caroline Dawson, l'auteure de l'AD présentée plus haut), d'un philologue, d'un traducteur ayant des connaissances dans le domaine de l'histoire de l'art et de l'AD, d'un artiste sensibilisé aux besoins des personnes handicapées ou d'une personne handicapée ayant une formation artistique ${ }^{7}$.

Les peintures ou les installations muséales peuvent être rendues accessibles aux personnes aveugles et malvoyantes grâce au travail commun d'un spécialiste en accessibilité, p. ex. de l'organisation Mind's Eye ou VocalEyes et d'un employé de musée. Généralement, lorsque la totalité ou une grande partie des collections est mise à la disposition du public, il s'agit d'un travail collectif auquel les personnes malvoyantes doivent sans doute aussi participer, conformément au principe « Rien pour nous, sans nous ").

En guise de conclusion, il faut remarquer que le type de tableau (abstrait ou figuratif) déterminera par conséquent la forme de la description. Les descriptions de tableaux abstraits peuvent donc constituer un défi, car ils ne présentent pas d'objets réels et jouent librement avec la forme et la couleur. C'est pourquoi c'est la narration qui aidera le visiteur à ordonner les informations. Dans l'ensemble, il n'y a pas de règle stricte de structuration de l'information, chaque image va en fait suggérer un ordre différent (cela exige que l'audiodescripteur réfléchisse à l'œuvre avant de pouvoir traduire correctement l'image en mots, en pratiquant le slow art). Je suis persuadée que les audiodescriptions devraient s'appuyer sur les résultats des recherches menées sur l'herméneutique de l'image ainsi que sur celles portant sur l'oculométrie, afin qu'une offre culturelle donnée soit réellement adaptée aux besoins des personnes malvoyantes.

${ }^{7}$ À Londres, c'est Lisa Squirrel de Barbican Art Gallery qui propose des visites avec l'AD, p. ex. Masculinities: Liberty Through Photography ou Into the Night: Cabarets and Clubs in Modern Art. 
Recherches menées dans le cadre du projet "Art accessible aux aveugles et aux malvoyants dans les musées de Londres " ("Accessible art for the blind and visually impaired in London museums ») du Programme Bekker de L'Agence Nationale Polonaise pour les Échanges Universitaires (Polish National Agency for Academic Exchange), $n^{\circ}$ PPN/BEK/2018/1/00150/U/00001. 


\section{BIBLIOGRAPHIE}

Ascott, R. (1998). Consciousness reframed: Art and consciousness in the post-biological era. Digital Creativity, 9 (1), 5-6. DOI : 10.1080/14626269808567099.

Boehm, G. (2014). O obrazach i widzeniu. Antologia tekstów. Kraków : TAiWPN Universitas.

Buswell, G.T. (1935). How People Look at Pictures: A Study of the Psychology of Perception in Art. Chicago : University of Chicago Press.

Chmiel, A. \& Mazur, I. (2014). Audiodeskrypcja. Poznań : Wydział Anglistyki UAM.

Coutrot, A., Binetti, N., Harrison, C., Mareschal, I. \& Johnston, A. (2016). Face exploration dynamics differentiate men and women. Journal of Vision, 16 (14), 1-19. DOI : 10.1167/16.14.16.

Fliescher, M. (2017). La pratique visuelle de l'Iconique. Regards croisés, 7, 45-54. Retrouvé sur : http:// hicsa.univ-paris1.fr/page.php?r=93\&id=592\&lang=fr.

Hutchinson, R. (2020). Museums for all: towards engaging, memorable museum experiences through inclusive audio description. Thèse de doctorat, London : University of Westminster.

Hutchinson, R. \& Eardley, A. (2018). Museum audio description: the problem of textual fidelity. Perspectives, 27 (1), 42-57. DOI : 10.1080/0907676X.2018.1473451.

Koide, N., Kubo, T., Nishida, S., Shibata, T. \& Ikeda, K. (2015). Art Expertise Reduces Influence of Visual Salience on Fixation in Viewing Abstract-Paintings. PLoS ONE, 10 (2), 1-14. DOI : 10.1371/ journal.pone.0117696.

Kołodziej, M., Majkowski, A., Francuz, P., Rak, R.J. \& Augustynowicz, P. (2018). Identifying experts in the field of visual arts using oculomotor signals. Journal of Eye Movement Research, 11 (3), 1-10. DOI : 10.16910/jemr.11.3.3.

Krejtz, K., Duchowski, A., Krejtz, I., Szarkowska, A. \& Kopacz, A. (2016). Discerning Ambient/Focal Attention with Coefficient K. ACM Transactions on Applied Perception, 13 (3), 1-20. DOI : $10.1145 / 2896452$.

Mazur, I. (2020). Audio Description: Concepts, Theories and Research Approaches. In Ł. Bogucki \& M. Deckert (eds.), The Palgrave Handbook of Audiovisual Translation and Media Accessibility (pp. 227-247). Cham : Palgrave Macmillan. DOI : 10.1007/978-3-030-42105-2.

Neves, J. (2012). Multi-sensory approaches to (audio)describing the visual arts. MonTI. Monografías de Traducción e Interpretación, 4, 277-293. DOI : 10.6035/MonTI.2012.4.12.

Randaccio, M. (2018). Museum Audio Description: Multimodal and 'Multisensory' Translation: A Case Study from the British Museum. Linguistics and Literature Studies, 6 (6), 285-297. DOI : 10.13189/ 1ls.2018.060604.

Snyder, J. (2007). Audio Description: The Visual Made Verbal. The International Journal of the Arts in Society: Annual Review, 2 (2), 99-104. DOI : 10.18848/1833-1866/CGP/v02i02/35358.

Sochoń, J. (1995). Hermeneutyka - wstępne rozpoznania. Warszawskie Studia Teologiczne, 8, 219-232.

Szarkowska, A., Jankowska, A., Krejtz, K. \& Kowalski, J. (2016). Open Art: Designing Accessible Content in a Multimedia Guide App for Visitors with and without Sensory Impairments. In A. Matamala \& P. Orero (eds.), Researching Audio Description (pp. 301-320). London : Palgrave Macmillan. DOI : 10.1057/978-1-137-56917-2_16.

Taylor, C. \& Perego, E. (2021). New approaches to accessibility and audio description in museum environments. In S. Braun \& K. Starr (eds.), Innovation in Audio Description Research (pp. 33-54). London/New York : Routledge.

Toury, G. (1986). Translation. A Cultural-Semiotic Perspective. In T.A. Sebeok (ed.), Encyclopedic Dictionary of Semiotics, 2 (pp. 1111-1124). Berlin : Mouton de Gruyter.

Vanmalderen, L. (1982). Un glossaire de la sémiologie de l'image. Communication et langages, 54 (4), 10-24. DOI : 10.3406/colan.1982.1516.

VocalEyes (n.d.). Audio Description and Access for Blind and Partially Sighted Visitors. Training manual for museum staff. London: VocalEyes. 